\title{
Review of aerosol delivery in the emergency department
}

\author{
Patricia A. Dailey ${ }^{1}$, Courtney M. Shockley ${ }^{2}$ \\ ${ }^{1}$ Department of Medical Affairs, Senior Medical Science Liaison, Galway, Ireland; ${ }^{2}$ Department of Emergency Medicine, Division of Pediatric \\ Emergency Medicine, UT Health San Antonio, San Antonio, TX, USA \\ Contributions: (I) Conception and design: PA Dailey; (II) Administrative support: Both authors; (III) Provision of study materials or patients: Both \\ authors; (IV) Collection and assembly of data: Both authors; (V) Data analysis and interpretation: Both authors; (VI) Manuscript writing: Both \\ authors; (VII) Final approval of manuscript: Both authors. \\ Correspondence to: Patricia A. Dailey. 112 Kimberly Drive, Manchester, CT 06040, USA. Email: PDailey@aerogen.com.
}

\begin{abstract}
Aerosol delivery is a vital therapeutic strategy for both adult and pediatric patients presenting to the emergency department with respiratory distress. Aerosolized bronchodilators are frequently used as rescue medications for patients with diagnoses of asthma, chronic obstructive pulmonary disease (COPD), or pneumonia. Historically, emergency department providers utilized jet nebulizers (JNs) for medication delivery, but were challenged by a need for increasingly higher bronchodilator doses to elicit the desired response. Advancements in technology have led to the development of newer specialized aerosol delivery devices and treatment strategies which provide clinicians with improved options for aerosol delivery but may also cause some uncertainty regarding appropriate device selection. Initial investigations comparing these devices presented valuable evidence of in vitro benefit but were unable to demonstrate corresponding improvement in clinical results. More recently there has been an influx of clinical evidence that suggests improved clinical outcomes associated with more efficient aerosol delivery devices such as vibrating mesh nebulizers (VMN) compared to the standard JN device. VMN will likely become an increasingly important tool in emergency department treatment of patients with respiratory distress. Additional controlled studies are needed both to examine the effects of VMN on patient outcomes, as well as to analyze how performance differences between aerosol devices may affect dosing strategies. Future efforts should also focus on applying new evidence in the form of updated consensus guidelines and standardized treatment strategies.
\end{abstract}

Keywords: Nebulizer; emergency department (ED); aerosol; jet nebulizer (JN); vibrating mesh nebulizer (VMN)

Submitted Jun 30, 2020. Accepted for publication Sep 20, 2020.

doi: $10.21037 / \mathrm{atm}-20-4724$

View this article at: http://dx.doi.org/10.21037/atm-20-4724

\section{Introduction}

Aerosol drug delivery is an essential component of effective care for patients presenting to the emergency department (ED) with respiratory distress, particularly those experiencing asthma or chronic obstructive pulmonary disease (COPD) exacerbations. Inhaled medications provide the benefit of a targeted delivery to the affected lung and airways while minimizing side effects associated with systemic absorption. Inhaled beta agonists are routinely administered to patients in respiratory distress due to their ability to rapidly provide relief of dyspnea, cough, and symptoms associated with acute airflow obstruction. In the
150 years, since their development, nebulized medications have become well-established as first-line treatments for patients with bronchoconstriction in both the ED and other acute care settings. Multiple aerosol delivery devices have been developed for use in the ED, including metered dose inhalers (MDI), jet nebulizers (JN) and vibrating mesh nebulizers (VMN). Clinicians unfamiliar with the components of effective aerosol delivery may find it difficult to select the most appropriate treatment device for each patient.

Multiple factors must be considered when selecting an aerosol delivery device for patients with respiratory distress. Optimal delivery of inhaled medications can be 
significantly affected by a patient's ability to perform proper inhalation technique, the interface of device to patient, their ability to use the device correctly, and the severity of airway obstruction. Familiarity with the basic operation, advantages, and disadvantages of each device, appreciation of clinical evidence comparing device performance, and an understanding of the resulting effects on clinical outcomes will facilitate appropriate device selection.

This review provides an overview of the function of aerosol delivery devices, summarizes available evidence supporting their utility in delivering inhaled medications to ED patients with respiratory distress, and examines new evidence comparing efficiency between aerosol delivery devices.

\section{JN}

The evolution of $\mathrm{JN}$ as a device of choice for inhaled medication delivery can be traced back to the mid 1800's with the introduction of apparatuses used to atomize liquids (1). Early versions of nebulizers used compressed air generated by a manual pump to exert pressure on a liquid, directing its flow against a baffle designed to break the liquid into respirable particles (1). In the early 1900's, inhaled epinephrine became the first aerosolized bronchodilator used for the treatment of asthma after published reports identified its use as an effective means of symptom control (1). As technology advanced through the mid $20^{\text {th }}$ century, both electric compressors and compressed oxygen were used to generate air flow to drive JNs. Although early modifications were made to shape, size and materials, since their origin JNs compressed gas to draw medication from a reservoir, shear liquid into particles and drive them into a baffle or wall of the nebulizer where larger particles impact and return to the reservoir, and smaller particles escape nebulizer when they can be inhaled by patients. These inhaled particles will not all reach the lower airways and lungs. Lung deposition of drug delivered by pneumatic $\mathrm{JN}$ is only a fraction of the total dose of drug placed in the nebulizer (termed "nominal dose") (2). Factors affecting JN performance and drug delivery include nebulizer design, driving gas flow and pressure, fill and residual volumes, gas density, and medication properties (solution, suspension, protein, peptide, etc.) (2). These and numerous other factors impact particle size, resulting in inconsistent device performance and medication delivery.

Despite their demonstrated utility in the acute care setting, there are disadvantages to using JNs to deliver inhaled medications. The compressed air or oxygen flow used to aerosolize liquid medications produces a continuous output of medical aerosol into the environment, creating potential for unintended drug delivery into a patient's eyes or inadvertent drug exposure to clinicians and caregivers (2). Additionally, JN reservoirs are susceptible to contamination through improper handling, oral secretions, or via the mouthpiece and may result in dispersion of bioaerosols $(3,4)$. While these devices are capable of aerosolizing liquid formulations, use with suspensions, proteins and peptides may be problematic. Residual volume ranges from 0.8 to $2 \mathrm{~mL}$, leaving as much as $2 / 3$ of the medication unused in the reservoir at the end of a treatment.

In an attempt to improve device performance, various alterations have been made to $\mathrm{JN}$ design, including the addition of a reservoir or collection bag, use of vents and valves and the development of both breath enhanced and breath actuated nebulizers. The addition of a reservoir or collection bag placed at the exit of the t-piece attachment with a series of one-way valves allows collection of aerosols exiting the nebulizer (2). This collected aerosol is then drawn from the reservoir providing an additional bolus of aerosol when the patient inhales. Breath enhanced nebulizers (BEN) utilize one-way valves to prevent the loss of aerosol into the environment and reroute aerosol to inspiration to increase medication delivery (2). Breath actuated JNs (BAN) produce aerosol only during inspiration. The patient must generate a minimum flow of $15 \mathrm{~L} / \mathrm{min}$ to trigger the aerosol producing mechanism and receive aerosol (2). Patients who are unable to generate sufficient flow to trigger the device will not receive any aerosol, which typically prompts the clinician to abandon use of the BAN in favor of a standard continuous output JN.

JN performance in aerosol delivery can vary widely between manufacturers, devices, and even between treatments using the same device. Inconsistent or inadequate device performance may negatively affect patient outcomes. Clinical comparisons of JN performance have not previously been feasible, as they were the only nebulizer commonly available for use in the acute care setting. With the advent of newer aerosol technology, it is imperative to explore whether the type of aerosol delivery device used may impact clinical outcomes such as medication dose requirement, airway responsiveness, ED length of stay (LOS), admission rate, and resource utilization. 


\section{JNs in the ED}

\section{FN vs. BAN $\mathcal{H N}$}

Several studies have sought to compare clinical performance of standard JN to breath actuated JN (BAN). In a single site, randomized comparative study, Parone and colleagues compared standard JN to BAN (AeroEclipse, Monaghan Medical) to determine which device utilized fewer treatments to resolve symptoms. The study's primary endpoint was total number of inhaled treatments. Secondary endpoints included peak expiratory flow rate (PEFR), respiratory rate, heart rate, and Modified Borg Score (a 0-10 rated scale used to assess dyspnea) $(5,6)$. Fiftyfour adult patients ( 27 in each treatment group) $>20$ years of age presenting to the ED with an Emergency Severity Index (ESI) triage level of 3 or 4 and a chief complaint of wheezing and dyspnea were enrolled in the study (6). The ESI is an emergency department triage score that stratifies patients into 5 groups based on severity, from level 1 (most severe) to level 5 (least severe) (7). Patients were randomized to treatment using either standard JN or BAN and received 1 to 3 nebulizer treatments (\#1 ipratropium bromide and albuterol sulfate, \#2 ipratropium bromide and albuterol sulfate, \#3 albuterol sulfate) in accordance with Advanced Nursing Guidelines in place at that institution. The authors found no statistically significant difference in either their primary endpoint (total number of treatments) or secondary endpoints (PEFR, respiratory rate, heart rate, and Modified Borg Score) between the standard JN and BAN. Furthermore, as use of BAN was associated with significantly longer treatment time compared to the standard JN group (46.8 vs. 33.7 minutes, respectively; $\mathrm{P}<0.02$ after 3 treatments) and incurred a nearly $90 \%$ increase in cost, the authors reported a lack of justification for their routine use.

In an RCT, Sabato et al. evaluated clinical outcomes in pediatric patients (0-18 years) who presented to the ED with asthma or wheezing (8). All patients were administered a single albuterol treatment in the ED via one of three different JNs: a BAN (AeroEclipse, Monaghan Medical), standard JN, or 1-hour of continuous JN. A total of 149 patients were enrolled with 84 patients randomized to BAN, 10 patients to small volume $\mathrm{JN}$, and 55 to continuous JN. Dosing was based on nebulizer used and patient weight. For patients randomized to BAN and small volume JN, those weighing $<20 \mathrm{~kg}$ received $0.5 \mathrm{~mL}(2.5 \mathrm{mg})$ albuterol in $0.5 \mathrm{~mL}$ normal saline (total solution $1.0 \mathrm{~mL}$ ) and those
$>20 \mathrm{~kg}$ received $(2.5 \mathrm{mg})$ albuterol undiluted (total solution $1.0 \mathrm{~mL}$ ). Continuous nebulization patients received $2.0 \mathrm{~mL}(10 \mathrm{mg})$ albuterol in $18 \mathrm{~mL}$ of normal saline (total solution $20 \mathrm{~mL}$ ) over 1 hour. Preferred interface for both BAN and small volume JNs was mouthpiece. An aerosol mask was used for all continuous JN and patients not able to coordinate mouthpiece (aerosol mask for standard and continuous JN, sealed mask for BAN). Blow by was used for patients unable to use mask. Both breath-actuated and continuous modes were used for BAN based on patient tolerance. There was no statistical significance reported between groups for the primary endpoint of ED LOS. Patients had significantly better improvement in clinical asthma score, a better decrease in respiratory rate, and a lower admission rate, and there was no significant difference in adverse effects. There were several limitations to this study that may have influenced the results. Selection of the patient interfaces affects aerosol delivery (9) and could influence patient outcomes. Several interfaces utilized with both small volume and continuous JN in this study such as open aerosol masks (9) and blow by (10) are less efficient interfaces for aerosol delivery compared to mouthpiece and closed mask interfaces utilized with the BAN. The American Association of Respiratory Care (AARC) recommends that the use of blow by should be abandoned (10). Another limiting factor is the inconsistent dosing and fill volumes used between the different devices. JNs do not function well with small fill volumes under $2 \mathrm{~mL}$ due to high residual volume (2), as used in this study. Required fill volumes for optimal performance of $\mathrm{JN}$ varies between devices (2) and total fill volume effects the amount of aerosol delivered (11).

\section{fNvs. BEN $7 N$}

There are a few ED studies comparing standard JN with BEN JN. Wilkinson et al. compared standard 1-hour long standard JN protocol with a "rapid albuterol delivery pathway" (12) using a BEN JN (NebuTech HDN, Salter Labs, Arvin, CA, USA) in 50 pediatric patients (ages 3 to 18) admitted to the ED with moderate to severe asthma determined by initial pediatric asthma score (PAS) (12). Patients were randomized to either a standard treatment arm with $\mathrm{JN}$ where they received up to 2 continuous hourlong albuterol nebulization (<20 kg; $10 \mathrm{mg}$ albuterol, $\geq 20 \mathrm{~kg} ; 15 \mathrm{mg}$ albuterol and $0.5 \mathrm{mg}$ ipratropium for both weights), or a rapid albuterol arm where they received up to 4 rapid albuterol treatments $(<20 \mathrm{~kg} ; 2.5 \mathrm{mg}$ albuterol, 
$\geq 20 \mathrm{~kg} ; 5.0 \mathrm{mg}$ albuterol and $0.5 \mathrm{mg}$ ipratropium for both weights) with a BEN, depending on severity scoring. Baseline and subsequent post treatment PAS were measured. The primary endpoint was ED LOS, measured by the start of randomization and ended at the time of disposition decision by the attending physician. There was no significant difference between ED LOS between groups (192 vs. 203 minutes, $\mathrm{P}=0.65$ ). They reported a significantly shorter study LOS (118 vs. 163 minutes, $\mathrm{P}=0.0002)$ which one could argue would be expected since required treatment times for the nebulizers studied were different (standard JN treatment arm; 1-hour long continuous and BEN treatment arm; shorter duration intermittent treatments, which are typically $<15$ minutes) (12). There are several other reported limitations of this study including lack of blinding. The final sample size was too small to make any definitive conclusions regarding secondary endpoints, including admission rates, revisit rates, changes in asthma score, and total albuterol dose (12).

Gardiner et al. completed an RCT in 107 pediatric patients 6 to 18 years of age presenting to the ED with acute asthma exacerbation and a forced expiratory volume 1 second (FEV1) $<70 \%$ of predicted, to compare treatment with JN (Hudson RCI Micro Mist nebulizer, Teleflex Medical, Research Triangle Park, New Jersey, USA) vs. BEN (NebuTech HDN, Salter Labs, Arvin, California, USA) (13). Primary outcome was change in FEV1, as measured by absolute improvement in percent of predicted and secondary outcomes included change in clinical asthma scores, ED LOS, disposition, and side effects (13). Baseline characteristics revealed that patients in the BEN arm were less commonly male, had lower baseline asthma severity, received more albuterol in the 24 hours prior to arrival to the ED, and a lower proportion of subjects who had received oral steroids prior to ED arrival. Results from this study demonstrated that albuterol delivered with a standard JN resulted in greater improvement in FEV1 than an equal treatment delivered by a BEN. There were no significant differences in secondary outcomes between groups including changes in clinical asthma scores (13). The authors report strengths of this study as successful blinding of study personnel enrolling and assessing patients, equal treatment of both groups and larger sample size compared to previous studies. In this study there was no demonstrated clinical advantage of using a BEN over a standard conventional JN in the treatment of acute exacerbations of pediatric asthma in the ED.

\section{MDls in the ED}

A MDI is a small hand-held drug/device combination that contains a specific drug. They are portable and provide low nominal dosages compared to other aerosol devices such as JN and VMN. Optimal aerosol delivery with an MDI requires a spacer or valved-holding chamber and is dependent on numerous factors such as patient/ clinician technique, coordination of the maneuver, shaking between puffs, timing of actuations with inspiration, breath hold, waiting 30 seconds between actuations, patient cooperation, ability to take a deep breath, and availability of the medication in the inhaler form (2). Inhalers come in a large variety of designs which can be confusing to both clinicians and patients and lead to sub-optimal aerosol delivery (2). With up to $60 \%$ of patients not using the MDIs to good effect, it is not uncommon that use of MDI at home provided little to no improvement prior to presentation to the ED. Doing more of the same in the ED may not provide added benefit. This can be a teachable moment for proper use of their MDI, but patients in respiratory distress have limited concentration and retention of new methods. This is the point when clinicians may want to consider another type of aerosol device with demonstrated clinical benefits.

There is an impressive amount of literature suggesting similar effectiveness with MDI with a valved-holding chamber compared to JNs $(14,15)$. Optimal administration with MDIs requires good hand-breath coordination and appropriate administration $(11,14,15)$. Patients who present to the ED in respiratory distress may be too distressed to perform effective maneuvers with an MDI, so the use of valved holding chambers should be considered. There may be a use for MDI in patients in the ED who present with mild symptoms and/or for those who require MDI training for use upon discharge to home. Moderate to severe exacerbations in patients with reversible airway disease and patients who are incapable of effectively using or responding to an MDI may respond better to aerosolized medication with nebulizer devices.

\section{VMN}

The VMN was introduced for the acute care setting in the early 2000s for the purpose of overcoming some of the disadvantages associated with common medical nebulizers. Active VMN use electricity to vibrate an aperture plate consisting of uniformly sized holes. Liquid sits on top of the 
plate and is drawn through the cone shaped holes which act as a micro-pump creating a mist of uniform droplets which results in consistent performance. There is no minimum fill volume, flow or pressure associated with VMN. Residual volume is less than $0.1 \mu \mathrm{m}$. While VMNs are available for use in ambulatory settings, the most common in the acute care setting is currently the Aerogen Solo (Aerogen Ltd., Galway Ireland). The nebulizer can be used with simple t-piece or for mouthpiece and aerosol mask interfaces with a handheld valved holding chamber adapter (Ultra, Aerogen Ltd., Galway, Ireland) that collects aerosol throughout the breathing cycle to optimize aerosol delivery to the patient. The VMN is separated from the mouthpiece and mask interface by valved-holding chamber which is not in communication with the reservoir cup and aperture plate that produces aerosol. This design prevents patient secretions from contaminating the reservoir cup and being aerosolized as bioaerosols (4). There is no flow generated with the VMN and therefore no active dispersing of aerosols. Scintigraphy studies have shown higher lung dose with VMN compared to JN (34.1\%, 5.2\%, respectively) $\mathrm{P}<0.001$ (16).

\section{VMN in the ED}

There have been multiple clinical studies in the ED comparing VMN with JN. The first was a large prospectively designed chart review study comparing use of $\mathrm{VMN}$ versus standard JN for patients of all ages [1,594] requiring short-acting bronchodilators (SABA) (17). This was a quality improvement plan with predetermined endpoints: admission rates, discharge rates, total amount of albuterol administered and ED LOS. Patients were included for two consecutive 30-day treatment periods. The first treatment period SABA ordered on all patients was administered using a standard JN per ED standard treatment protocol. The second treatment period SABA ordered on all patients was administered using a VMN per ED standard treatment protocol. There were 879 patients recruited in the JN group and 715 in the VMN group. Baseline demographic data was similar between groups. Admission rate was significantly lower for all patients treated with VMN (13.3\% absolute, 32\% relative) compared to patients treated with JN $(\mathrm{P}<0.05)$. The total albuterol dose administered was significantly lower in the VMN group $(\mathrm{P}<0.001)$. Eighty-five percent of patients treated with $\mathrm{VMN}$ responded to a single $2.5 \mathrm{mg}$ albuterol dose compared to $47 \%$ of patients treated with
JN. No patients in the VMN group required more than 5.0 $\mathrm{mg}$ albuterol, while $24 \%$ of patients in JN group required $>7.5 \mathrm{mg}$ albuterol with some needing continuous albuterol. There was also a 37-minute median decrease in the ED LOS in the VMN group compared to the JN group, a $13 \%$ reduction $(\mathrm{P}=0.0001)$. They also reported that patients 65 years and older had lower admission rate in the VMN group compared to the JN group (61\% vs. $76 \%$, respectively). The authors concluded that device type was a strong predictor of discharge, disposition, LOS and total amount of drug, regardless of age or diagnosis (17). While compelling, RCT clinical studies specific to diagnosis and patient population should be done to corroborate these results.

Moody et al. reported a single-blind randomized clinical trial of 217 children (2-18 years of age) with a moderate to severe asthma exacerbation in the ED (18). The aim of the study was to compare clinical outcomes associated with the use of VMN or JN with either mouthpiece or mask interface for the delivery of inhaled bronchodilators in the treatment of acute asthma exacerbations in the ED. Patients were randomized to JN [109] with mouthpiece or dragon mask or VMN [108] with mouthpiece or valved-mask. Asthma severity score was assessed utilizing a pediatric assessment score (PAS) that rated respiratory rate, oxygen requirement, retractions, and auscultation. Patients were categorized as either mild: $1-4$, moderate: $5-8$ or severe: 9-12. Baseline assessment and PAS were performed by the treating respiratory therapist (18). Treatment regime used in this study for moderate to severe PAS consisted of 3 treatments with albuterol and ipratropium over 20-min increments. Post treatment assessments were completed 15-20 min after initiation of treatment to ensure blinding of the investigator and every 20 -min if the patient did not receive another treatment. The study was stopped when disposition decision was made by treating physician. Decision to admit, discharge and/or provide additional treatments despite achievement of a mild asthma score was at the sole discretion of the treating physician. The primary endpoint was admission rates. The median baseline PAS was significantly higher in the VMN group (9.0 vs. 8.0) $(\mathrm{P}=042)$, otherwise baseline demographics were similar between groups. A $31 \%$ lower admission rate $(6.3 \%)$ in the VMN group was clinically meaningful but not statistically significant $(\mathrm{P}=0.22)$, possibly an effect of sample size. This could have been an effect of the sample size and limitation of the study. Adjustment for baseline difference in PAS and weight showed that patients treated with the VMN had a higher probability of being discharged overall 
$(\mathrm{P}=0.062)$. Stratification by interface (mouthpiece $v s$. mask) demonstrated that patients treated with the VMN and mask showed a $48 \%$ relative (10\% absolute) lower admission rate compared to patients treated with JN and mask $(\mathrm{P}=0.07)$. Adjustment of baseline AS using a logistic regression model showed that VMN combined with valved-mask significantly reduced the probability of admission compared to JN with aerosol mask $(\mathrm{P}=0.032)$ (18). This implies that both selection of mask interface and nebulizer are an important predictor of clinical outcomes in acute moderate to severe exacerbations in pediatric asthma patients in the ED. The time to achieve a mild PAS regardless of severity level was significantly shorter in the VMN group compared to the JN group ( $\mathrm{P}=0.004)$. Stratification by severity showed significantly shorter time to mild score in the VMN group $v s$. JN group for both moderate and severe scores $(\mathrm{P}=0.01$, $\mathrm{P}=0.02$, respectively). Faster resolution of symptoms could allow physicians to make critical decisions on treatment pathways and disposition decisions. The median number of treatments required to achieve a mild asthma score was significantly less in the VMN group than in the JN group $(\mathrm{P}<0.001)$, regardless of baseline asthma score (18). Patients treated with VMN compared to $\mathrm{JN}$ in this study demonstrated lower admission rates, reduced probability of admission, significantly fewer treatments to mild asthma score and significantly less time to achieve a mild asthma score compared to JN. This study highlights the importance of clinician awareness on the appropriate selection of aerosol delivery device and interface type and their impact on clinical outcomes.

Cantu and Jenkins presented results from a large prospectively designed chart review study for the purpose of comparing albuterol delivery with large volume JN $v s$. VMN to determine the effect on clinical outcome in pediatric patients (ages 2 to 18 ) with acute exacerbation of asthma and reactive airway disease (RAD) in the ED (19). This quality improvement plan compared 90-day treatments periods for two consecutive years (December and March 2018 and 2019). Predetermined endpoints included the total mean dose of albuterol $(\mathrm{mg})$ administered (primary outcome), total number of treatments, ED LOS before discharge or disposition, admission rate, and 48-hour ED readmission rate (secondary outcomes). In the first 90-day treatment period (December 2017-March 2018) all patients were treated with a large volume JN (Airlife Misty Finity Large Volume Nebulizer, Carefusion, Yorba Linda, CA, USA) using a weight-based treatment protocol $(<20 \mathrm{~kg} ; 2.5 \mathrm{mg}$ albuterol and $>20 \mathrm{~kg}$ :
$5 \mathrm{mg}$ albuterol). In the second 90-day treatment period (December 2018-March 2019) all patients were treated with a VMN with a valved-holding chamber (Aerogen Solo with Ultra, Aerogen Ltd., Galway, Ireland) with $2.5 \mathrm{mg}$ albuterol $(<20 \mathrm{~kg})$ received and $5 \mathrm{mg}$ albuterol $(>20 \mathrm{~kg})$. Data extracted from the electronic medical record (EMR; MedTech) revealed that 608 patients in the JN group with a mean age $6.24 \pm 3.64$ years in the first year. In the second year there were 537 patients with a mean age $6.43 \pm 3.81$ years. Total mean dose of albuterol was less in the VMN group compared to the large volume JN group $(3.72 \pm 1.28 v s$. $11.23 \pm 5.21 \mathrm{mg}$, respectively) $(\mathrm{P}<0.001)$. In addition, fewer treatments were administered with intermittent $\mathrm{VMN}$ $v s$. hour long large volume $\mathrm{JN}$ treatments $(1.45 \pm 0.64 v s$. $1.52 \pm 0.72$, respectively $)(\mathrm{P}<0.001)$. The decrease in the amount of albuterol and number of treatments required with VMN vs. JN are consistent with results observed in the two previous studies comparing VMN and JN $(17,18)$. In addition, admission rates associated with $\mathrm{VMN} v s$. JN was reduced by $41 \%$. This was consistent with observations by Dunne \& Shortt (32\%) and Moody et al. $(31 \%)(17,18)$.

This study also observed a decrease in the ED LOS by an average of 80 minutes (19). Decreasing time in the ED is important for both the patient and clinician. Less time in the ED means improved throughput allowing for treatment of more critically ill patients and faster discharge of patients who recover from their exacerbation.

Chweich et al. presented results from a single-blinded, parallel, single centered RCT in severe asthma exacerbation in adults, defined as PEFR $<50 \%$ of predicted in patients in the ED (20). Patients were randomized to standard JN or VMN (Aerogen Solo with Ultra, Aerogen Ltd., Galway, Ireland) and treated using the existing ED asthma protocol. For the 31 patients ( $16 \mathrm{JN}, 15 \mathrm{VMN}$ ). There was a trend to greater change in mean PEFR\% from baseline for VMN vs. JN group (23.3 vs. 14.4, respectively) $(\mathrm{P}=0.063)$ with greater change in mean FEV1\% with VMN vs. JN group (17.1 vs. 8.0, respectively) $(\mathrm{P}=0.045)(20)$. The endpoints in this study were unique compared to some of the other clinical outcome ED comparisons studies in this review. Airflow measurements can provide the clinician with additional information regarding severity of airflow obstruction and are a useful tool. However, these measurements may be challenging in patients with severe exacerbations due to difficulty in performing maneuvers during distress. This study provides us with some insightful data suggesting faster improvement sustained over time in severe asthma exacerbations with $\mathrm{VMN} v s$. JN. 
One ED reported that their experience with limited clinical response to standard dosing of short acting bronchodilators resulted in the need for use of hour-long aerosol therapy with $\mathrm{JN}$ to accommodate the required high dosages for asthma and COPD patients treated in their ED (21). To determine how to reduce the amount of albuterol administered to patients admitted to the ED for exacerbations, a prospective chart review quality improvement project was conducted in COPD and asthma patients presenting to the ED with breathlessness requiring bronchodilator therapy (at the treating physician's discretion (21). There were two treatment periods. During period 1 patients were treated per the ED treatment protocol with JN and period 2 with VMN. Data was extracted from the EMR for 199 patients $(118 \mathrm{JN}, 81$ VMN) between December 10, 2018 and April 5, 2019. They demonstrated significantly less total mean albuterol $(\mathrm{mg})$ in the VMN group compared to $\mathrm{JN}$ group $(7.9 \pm 5.4$ vs. 13.8 \pm 9.6 , respectively) $(\mathrm{P}=0.0003)$ and total treatment time (minutes) with the VMN group was significantly less compared to the JN group $(40.7 \pm 24.9$ vs. $63.1 \pm 40.4$, respectively) $(\mathrm{P}=0.002)$ for asthma patients. They also reported significantly fewer treatments for asthma and COPD combined with $\mathrm{VMN} v s$. JN $(2.1 \pm 1.6$ vs. $2.7 \pm 1.6$. respectively) $(\mathrm{P}=0.009)$. In asthma, 1 out of $42(2.4 \%) \mathrm{VMN}$ required an hour-long treatment compared to 30 out of 63 $(47.6 \%)$ in the JN group $(\mathrm{P}<0.001)$ while in COPD patients 0 of the $39 \mathrm{VMN}$ required hour-long treatments compared to 9 out of $55(16.4 \%)$ in the JN group $(\mathrm{P}<0.001)$. The higher doses and longer treatment times are associated with the use of hour-long therapy with large volume JN. Based on these findings the investigators were able to reduce the number of hour-long treatments, and amount of albuterol overall with VMN. This reduction in amount of albuterol and number of treatments are consistent with other studies comparing $\mathrm{VMN}$ to JN for aerosol delivery in the ED (17-19).

\section{Dosing strategies}

Prompt administration of an inhaled short acting beta agonist (SABA) medication, most commonly albuterol, is firmly established as a mainstay of therapy for ED management of acute airflow obstruction. Albuterol exerts its pulmonary effects through its action on $\beta 2$-adrenergic receptors of the smooth muscle in the lungs. An intracellular cascade of events results in relaxation of smooth muscle in the trachea and bronchi, serving to ease bronchospasm and improve airway resistance (22). Stimulation of $\beta$-adrenergic receptors may also produce unfavorable effects, including excitability, tremor, sinus tachycardia, QT prolongation, supraventricular tachycardia, nausea, vomiting, and hypokalemia (22). As is true for any therapy, dosing strategies should strive to maximize symptom improvement while minimizing potential adverse effects.

Drug manufacturers' recommendations for administration typically reflect the minimum quantity and frequency of medication required to improve airway obstruction while minimizing adverse effects in a stable patient with moderate disease. Conversely, clinical guidelines formulated by expert consensus, such as those provided by the National Institutes of Health (NIH) often advocate for a more aggressive treatment approach than is suggested by the manufacturer (23). Current evidence and consensus guidelines suggest that the optimal dose of albuterol for ED treatment of severe asthma is between 1.2 and $2.4 \mathrm{mg} / \mathrm{h}$ (4-8 puffs or $2.5-5.0 \mathrm{mg}$ every 20 minutes) when given via MDI and between 2.5 and $15 \mathrm{mg} / \mathrm{h}$ (or 0.15 to $0.3 \mathrm{mg} / \mathrm{kg}$ up to $10 \mathrm{mg}$ for children $<12$ years of age) when administered via nebulizer $(22,23)$. Similarly, both the Food and Drug Administration (FDA) and National Asthma Education and Prevention Program (NAEPP) recommend initial therapy with $2.5-5.0 \mathrm{mg}$ every 20 minutes for 3 treatments (22). Although the benefit of aerosolized SABA in treating acute bronchoconstriction is largely undisputed, considerable disparities exist in terms of medication dosing and treatment frequency. Many institutions have developed their own approach to bronchodilator treatment protocols $(8,24)$. Results of a 2011 questionnaire distributed to respiratory care directors of 43 children's hospitals (Child Health Corporation of America), revealed that among respondents, $76 \%$ (16 of 21 ) relied upon institutional protocol to guide albuterol dosing, and 60\% (6 of 10) endorsed using doses above current guidelines (24).

When symptoms of acute severe airway obstruction fail to adequately improve with standard doses of bronchodilators, clinicians must re-evaluate their strategy for SABA resuscitation. Commonly, this approach seeks to improve lower respiratory tract deposition through an increase in the dose and/or frequency of inhaled medications $(23,25)$. Although evidence regarding effects of albuterol administered at doses of 2.5 to $15 \mathrm{mg} /$ hour is conflicting, it is widely believed that higher cumulative dosages may be required to improve pulmonary function in patients with more severe disease (12). McFadden and colleagues evaluated the bronchodilator effects of two $5 \mathrm{mg}$ doses (high dose) of aerosolized albuterol administered 
over 40 minutes compared to three $2.5 \mathrm{mg}$ doses (standard dose) administered over 60 minutes. One hundred and sixty patients presenting to the ED with an acute asthma exacerbation were enrolled in a prospective sequential design. Although both regimens improved Peak Expiratory Flow, the authors concluded that the high dose regimen improved lung function more rapidly and to a larger extent than the standard dose (26). The same investigators then performed a randomized double-blind study comparing the efficacy of high and low dose albuterol administered to asthma patients in the ED. Nebulized albuterol of either $7.5 \mathrm{mg}$ (high dose) or $2.5 \mathrm{mg}$ (low dose) was administered every 20 minutes for 3 doses to 160 patients. The authors reported no significant difference between groups in either FEV1 (50.3\% vs. $44.6 \%)$ or admission rate (43\% vs. 39\%) and concluded there was no clinical advantage to the use of $7.5 \mathrm{mg}$ when compared to the $2.5 \mathrm{mg}$ dose (27). In 2016, a large double-blind multicenter study was conducted to assess the effect of increased albuterol dosage regimens on ED LOS and hospital admission rate. The authors enrolled patients between 2-17 years of age presenting to the ED with acute moderate to severe asthma. The control group received 6 (weight $<25 \mathrm{~kg}$ ) or 12 puffs (weight $>25 \mathrm{~kg}$ ) while the study group was given 9 (weight up to $15 \mathrm{~kg}$ ), 12 (weight $>15-20 \mathrm{~kg}$ ), 15 (weight $>20-25 \mathrm{~kg}$ ), or 18 puffs (weight $>25 \mathrm{~kg}$ ). One hundred and nineteen patients were included in the study, and no significant difference was found either in LOS $(\mathrm{P}=0.48)$ or admission rate $(\mathrm{P}=0.55)$. There were also no significant differences observed in FEV1, clinical respiratory score, or pulse oximetry changes, leading the authors to conclude that while higher albuterol dosage regimens failed to shorten ED LOS or lower admission rate, they demonstrated a similar safety profile within the study population (28).

Inconsistent demonstration of improved clinical outcomes using higher $v s$. standard albuterol treatment doses may be attributable not only to the medication, but also to individual patient factors. Severe airway inflammation and obstruction may induce a state of refractoriness to aerosol therapy, thereby limiting response to increased dose and frequency of bronchodilator medication (23). Furthermore, severe bronchoconstriction may cause preferential drug deposition at sites of airway narrowing, preventing medication delivery to the target sites of action (23). In a prospective, sequential evaluation of 116 patients presenting to the ED with acute exacerbations of asthma, Rodrigo and Rodrigo examined therapeutic response patterns and sought to identify factors that may contribute to clinical outcome. All patients were given 4 puffs of albuterol via MDI with spacer at 10 min intervals for 3 hours. Although all patients demonstrated a dose related improvement in PEFR, subgroup analyses revealed that $30 \%$ of patients did not respond sufficiently and required hospital admission. Notably, the majority of patients who demonstrated a favorable response required $<2.4 \mathrm{mg}$ of albuterol to reach discharge criteria, whereas in patients requiring admission high doses of albuterol had minimal effect. The most important predictors of outcome within the study population were not PEFR or PEFR percent of predicted obtained on initial evaluation, but rather PEFR, PEFR percent of predicted, and PEFR improvement over baseline measured at $30 \mathrm{~min}$ (29). The authors proposed that early, short-term treatment response, rather than initial symptom severity, was the most important predictor of clinical outcome, a conclusion that suggests early response to albuterol may reasonably predict requirement for hospital admission in patients with severe asthma (23). Similarly, Strauss et al. monitored the PEFR of 92 acutely ill adult patients who received $2.5 \mathrm{mg}$ of nebulized albuterol every 20 minutes for 3 doses. As in Rodrigo's study, nearly $70 \%$ of enrolled patients had sufficient improvement in PEFR were safely discharged from the ED, and required $\leq 5 \mathrm{mg}$ of albuterol to reach discharge threshold. The remaining $30 \%$ failed to demonstrate PEFR improvement $>40 \%$ of expected value despite receiving $7.5 \mathrm{mg}$ of nebulized albuterol (30). Most recently, investigators at Tufts Medical Center performed a prospective, single blind pilot study comparing clinical response between adult asthmatics treated with VMN vs. JN. Although the sample size was small $(n=31)$ the authors reported a clinically meaningful and significant difference respectively in the PEFR $(\mathrm{P}=0.063)$ and FEV1 $(\mathrm{P}=0.045)$ values obtained after $30 \mathrm{~min}$ of nebulized aerosol treatment (20). The more rapid improvement noted in patients utilizing a VMN device was sustained through ED discharge, again suggesting that a patient's initial response to bronchodilator treatment is a valuable indicator of favorable outcome.

For patients in whom aggressive initial bronchodilator therapy fails to produce adequate symptom relief, continuous nebulization may offer a less labor-intensive and more cost-effective treatment strategy. Multiple randomized clinical trials have evaluated the risk versus benefit profile of continuous versus intermittent administration of bronchodilators in adult and pediatric patients with moderate to severe respiratory distress. While reports of enhanced clinical outcome with continuous 
compared to intermittent therapy are mixed, several studies describe either equivalent or reduced incidence of adverse events (tremor, tachycardia, hypertension, hypokalemia) and discrete advantages in clinician time spent administering therapy when medications are administered continuously (28,31-33).

Often overlooked in these discussions, however, is the potential for paradoxical bronchoconstriction that may occur with the use of continuously nebulized albuterol. Owing to higher dose requirements and larger volumes of albuterol necessitated by continuous treatments, many institutions prepare multidose vials that contain benzalkonium chloride (BAC) as a preservative (34-36) BAC has been demonstrated to have a potent, cumulative, and prolonged negative effect on airway responsiveness (34). Orth and colleagues retrospectively reviewed the records of 128 patients who received continuous nebulized albuterol (CNA) therapy and found no significant difference in the median duration of CNA $(\mathrm{P}=0.19)$, maximum albuterol dosing requirement $(\mathrm{P}=0.89)$, or median duration of supplemental oxygen use $(\mathrm{P}=0.77)$ between cohorts exposed $(n=80)$ or not exposed $(n=48)$ to BAC (35). Conversely, a more recent and much larger retrospective cohort study comparing clinical outcomes between patients $<18$ years of age exposed to BAC $(n=236)$ versus not exposed to BAC $(n=241)$ concluded that BAC antagonized the function and effect of nebulized albuterol (36). In this study population, the authors noted a $50 \%$ longer duration of CNA in those exposed to BAC than in those not exposed (median treatment time of $9 v s .6$ hours, respectively). Additionally, patients not exposed to BAC were $79 \%$ more likely to be weaned from CNA (hazard ratio $1.79 ; 95 \%$ CI: 1.45 to 2.22; $\mathrm{P}<0.001$ ) and $43 \%$ more likely to be weaned from additional respiratory support (hazard ratio $1.43 ; 95 \%$ CI: 1.16 to $1.75 ; \mathrm{P}<0.001)$ (36). Furthermore, research presented by investigators at Norton Children's Hospital in Louisville, KY suggests that BAC may be wholly unnecessary to maintain either the stability or sterility of multi-dose albuterol vials. When BAC containing and preservative free $(\mathrm{PF})$ albuterol solutions prepared in both high $(0.67 \mathrm{mg} / \mathrm{mL})$ and low $(0.17 \mathrm{mg} / \mathrm{mL})$ formulations were stored at room temperature and under refrigeration, high performance liquid chromatography demonstrated no difference in stability of either formulation for up to 7 days. In addition, after 10 days of incubation in both aerobic and anaerobic media, no bacterial growth was demonstrated in either BAC containing or PF albuterol solutions (34). With the aforementioned potential of BAC to increase risk of poor treatment response without affording any measurable benefit in maintaining stability or sterility, clinicians choosing to administer CAN treatment should strongly consider using PF formulations.

Despite having a different mode of action and slower onset of action than $\beta$-agonists, inhaled short-acting anticholinergic (SAAC) agents are often used to enhance and prolong bronchodilation achieved with inhaled $\beta$-agonists $(37,38)$. Ipratropium inhibits cholinergic receptors in the airways, decreasing contractility of bronchial smooth muscle and reducing mucosal edema and secretions $(37,38)$. Two large meta-analyses recently examined the capacity of combined treatment with SABA and inhaled SAAC to improve lung function and reduce hospital admission in patients with acute asthma exacerbations. In 2017, Kirkland et al. performed a systematic review of 23 studies including 2,724 patients (majority $\geq 16$ years or age) presenting to the ED with acute asthma. Overall, combination inhaled therapy with both SAAC and SABA more effectively reduced hospital admission than inhaled therapy with SABA alone (RR 0.72; $95 \%$ CI, 0.59 to $0.87 ; 16$ studies, 2,120 participants). Approximately 65 fewer patients per 1,000 would require hospital admission after receiving combination inhaled therapy (95\% CI, 30 to 95), compared to 231 per 1,000 patients receiving inhaled therapy with SABA alone. Although the quality of evidence was not as robust, patients receiving combination inhaled therapy were also found to be more likely to attain improvements in FEV1, PEFR, and PEFR increase above baseline, and were less likely to require additional ED visits for further care. In contrast to previously reported data, the authors did note that study participants treated with combination inhaled therapy demonstrated a higher incidence of adverse effects (tremor, agitation, and palpitations) than those treated with inhaled SABA alone (OR 2.03; 95\% CI, 1.28 to 3.20, 1,392 participants, 11 studies) (39). A 2013 Cochrane meta-analysis of 24 comparison studies including 2,697 children treated in the ED for moderate or severe asthma exacerbations concluded that children treated with combination inhaled therapy were significantly less likely to require hospital admission (RR $0.73 ; 95 \%$ CI, 0.63 to $0.85 ; 15$ studies, high quality evidence) than those treated with inhaled SABA alone and reported an overall number needed to treat (NNT) of 16 (95\% CI, 12 to 29) (37). As in the review conducted by Kirkland et al., there was no significant difference noted in regard to ED return rates between groups. Unlike the findings reported by Kirkland et al., however, Griffiths et al. reported a lower incidence 
of adverse effects (nausea and tremor) in children treated with combination inhaled therapy than in those than those treated with inhaled SABA alone $(37,39)$.

\section{Implications of new technology on dosing strategies}

Thus far, dosing recommendations for inhaled medications have been established using data on the lung deposition that is achieved with the use of standard JN, enhanced JN (BAN or BEN) or MDI. When challenged by patients demonstrating inadequate symptom relief with standard bronchodilator therapy, clinicians commonly attempt to enhance drug deposition by intensifying either the total dose and/or frequency of inhaled treatments. Data presented in prior sections of this review support an alternative strategy to enhance lung deposition of inhaled medications, not by increasing the dose or frequency of therapy, but rather by employing more efficient aerosol technology. Briefly, statistically significant clinical outcomes attained with the use of $\mathrm{VMN}$ as compared to JN included: reduction in total albuterol dose (Dunne and Shortt, $\mathrm{P}<0.05$; Moody $\mathrm{P}<0.001$; Cantu $\mathrm{P}<0.001$; Carragher $\mathrm{P}=0.0003)$, decrease in total number of albuterol treatments (Cantu $\mathrm{P}<0.001$; Carragher $\mathrm{P}=0.009$, Moody $\mathrm{P}<0.001)$, shorter CNA total treatment time (Carragher $\mathrm{P}=0.002$ ), improved mean $\mathrm{PEFR} \%$ and FEV $1 \%$ change from baseline (Chewich $\mathrm{P}=0.063$ and $\mathrm{P}=0.045$, respectively), and reduction in total ipratropium dose (Moody $\mathrm{P}<0.001)(17-21)$.

Although encouraging, it is crucial to recognize that these studies primarily sought to compare the effect of aerosol delivery devices; medication doses, therapy duration, and improved lung function which were utilized as outcome measures. To date, no studies have compared the efficacy, adverse effects, or clinical outcomes between varying doses of SABA, SAAC, or combination inhaled therapy when administered with VMN. With the recent emergence of in vitro and clinical data demonstrating superior lung deposition with the use of $\mathrm{VMN}$, it becomes imperative to re-examine dosing strategies to reflect the increased efficiency of new aerosol technology. Use of a more efficient aerosol delivery device to treat patients with acute bronchoconstriction may allow dosing strategies that more closely align with recommendations set forth by drug manufacturers, potentially reducing the risk of adverse medication effects, as well as exposure to potentially bronchoconstrictive preservatives.

\section{Discussion}

For decades, ED providers have utilized JN to deliver aerosol medications to patients with acute exacerbations of asthma or COPD. Over time, the inefficiencies associated with JN have been made apparent by a need for increasingly higher or more frequent bronchodilator doses to elicit the desired therapeutic response. Many institutions have sought to overcome this challenge through the use of high dose bronchodilators, back to back intermittent treatments, or continuous aerosol delivery with small or large volume JN. The remarkable growth that has occurred in aerosol delivery technology may cause confusion regarding appropriate device selection.

Fortunately, new evidence demonstrates improved clinical outcomes associated with the use of more efficient aerosol delivery devices. Although limited data supports the use of specialized JN such as BAN or BEN over standard $\mathrm{JN}$, this evidence is often conflicting and not consistently reproduced between studies. Additionally, since a large body of literature suggests equivalence between the use of standard JN and MDI with VHC, nebulizers that demonstrate better clinical outcomes than standard JN may also produce better clinical benefits than MDI. Recent evidence in favor of the use of VMN compared to standard $\mathrm{JN}$ provides compelling data that is consistently replicated between studies. In multiple studies and across a variety of outcomes, standard JN failed to perform as well as VMN in regard to albuterol dose and number of treatments required, aerosol treatment times, patient airflow indices, time to achieve mild asthma scores, ED LOS, and requirement for hospital admission.

While existing evidence clearly indicates advantages of VMN technology, several variables should be further investigated, and additional benefits explored. Data presented in both the Carragher and Cantu studies demonstrate that when compared to JN, use of VMN reduces both the total albuterol dose required, as well as the treatment time for patients with acute asthma exacerbation $(19,21)$. Both scintigraphy studies and clinical data have shown superior lung deposition with use of VMN, however, as discussed by Moody, clinicians may question whether this benefit can be overcome by using higher doses of nebulized albuterol through a standard JN (18). New studies should aim to evaluate the correlation between device performance, dose-equivalence, and clinical outcomes (18). Moody study found a reduction in the amount of inhaled ipratropium required when delivered via VMN (18). Although an 
inhaled route of administration reduces systemic side effects, it cannot entirely eliminate the risk. Enhanced drug delivery and potential for exposure to lower doses of adjunct medications may further decrease the risk of drug side effects. Future investigations should assess for discrepancies in adverse events based on the device used for aerosol delivery.

New clinical trials should be designed to stratify study population according to exacerbation severity, as VMN may provide the most robust improvement in patients with severe symptoms. Dunne and Shortt found no significant difference in hospital admission of patients 3-18 years of age based on device used, however, a requirement for more than $5 \mathrm{mg}$ of albuterol in only a small minority of patients (23\% in JN group, $0 \%$ in VMN group) likely reflects lower disease severity within the study population $(17,18)$. Conversely, Moody observed an absolute reduction in hospital admission with VMN when results were analyzed by exacerbation severity ( $7.2 \%$ for moderate, $8.4 \%$ for severe) (18). While not able to achieve statistical significance due to limitations described previously, this data is certainly clinically meaningful. Exacerbation severity may also influence concerns regarding device function and proper use. As discussed in previous sections, correct technique is essential for optimal device function. Moderate or severe respiratory distress may impair handbreath coordination and prohibit a patient from effectively performing MDI maneuvers. Furthermore, patients who require escalation of care to continuous nebulized therapy may be better served by use of VMN. While both MDI and nebulizers are capable of delivering intermittent treatments, continuous aerosol therapy must be administered with a nebulizer. Additional randomized controlled trials may help clarify the impact of $\mathrm{VMN}$ on varying levels of exacerbation severity, enabling clinicians to identify the patients most able to benefit from VMN use.

Proper device technique relies upon multiple factors, each of which presents added opportunity for further study. Even when exacerbation severity does not impede proper technique, a patient's age or cognitive ability may influence their ability to comply with instructions. Clinicians and caregivers must also be proficient in operation of the chosen device in order to appropriately instruct and assist in correct use. Some institutions may be hesitant to incorporate new aerosol technology into treatment protocols due to concerns regarding device usability. To date, no studies have been performed comparing the ease of use between MDI, JN, and VMN either overall or among different ages or levels of cognitive ability. Device interface should also be more thoroughly investigated, as there is no clear consensus on whether mouthpiece or face mask offers superior drug delivery when combined with VMN. Though some evidence appears to support more efficient delivery with use of a mouthpiece, other data has found enhanced delivery when mask interface is used (18). Correct technique remains essential, regardless of interface used. If future investigations aim to establish superiority of either the mouthpiece or mask interface when used with VMN, subjects should be carefully supervised to ensure correct technique.

Finally, in the modern healthcare landscape, clinicians must be mindful of healthcare costs, resource utilization, and patient/caregiver satisfaction. Some would contend that the potential to effectively treat acute respiratory distress with lower drug doses, shorter treatment durations, and fewer adjunct agents, as well as the potential to reduce hospital admission rates and more efficiently utilize resources may offset the increased expense typically associated with newer technology. Going forward, formal analyses of cost-effectiveness are needed. Additional data should also be collected comparing patient/caregiver satisfaction level between each method of aerosol delivery to determine whether use of a more efficient device can improve patient experience.

As the technology for inhaled drug delivery continues to advance, efforts should be made to periodically review and apply best evidence to update consensus guidelines and standardize treatment strategies. More randomized controlled trials are needed to comprehensively evaluate respiratory populations and diagnoses that require aerosol delivery in the ED.

\section{Acknowledgments}

Funding: None.

\section{Footnote}

Provenance and Peer Review: This article was commissioned by the Guest Editors (Dr. James B. Fink and Dr. Zhe Luo) for the series "Medical Aerosol in Acute and Critical Care" published in Annals of Translational Medicine. The article has undergone external peer review.

Conflicts of Interest: Both authors have completed the ICMJE uniform disclosure form (available at http://dx.doi. 
org/10.21037/atm-20-4724). The series "Medical Aerosol in Acute and Critical Care" was commissioned by the editorial office without any funding or sponsorship. Dr. PAD reports other from Aerogen Ltd., outside the submitted work. The authors have no other conflicts of interest to declare.

Ethical Statement: The authors are accountable for all aspects of the work in ensuring that questions related to the accuracy or integrity of any part of the work are appropriately investigated and resolved.

Open Access Statement: This is an Open Access article distributed in accordance with the Creative Commons Attribution-NonCommercial-NoDerivs 4.0 International License (CC BY-NC-ND 4.0), which permits the noncommercial replication and distribution of the article with the strict proviso that no changes or edits are made and the original work is properly cited (including links to both the formal publication through the relevant DOI and the license). See: https://creativecommons.org/licenses/by-nc-nd/4.0/.

\section{References}

1. Nikander K, Sanders. The early evolution of nebulizers. Available online: http://incenter.medical.philips.com/ doclib/enc/fetch/2000/4504/577242/577256/ 588821/5050628/5313460/7063104/12_MM_54-3_ Nicander.pdf\%3fnodeid\%3d7061353\%26vernum\%3d1

2. Gardenhire DS, Burnett D, Strickland S, et al. A Guide to Aerosol Delivery Devices for Respiratory Therapists. Available online: https://www.aarc.org/wp-content/ uploads/2015/04/aerosol_guide_rt.pdf

3. Dolovich MB, Ahrens RC, Hess DR, et al. Device selection and outcomes of aerosol therapy: Evidencebased guidelines: American College of Chest Physicians/ American College of Asthma, Allergy, and Immunology. Chest 2005;127:335-71.

4. Dailey P, Fink JB. Aerosol Contamination Associated with Simulated 'Drooling' Into a Nebulizer Mouthpiece. ISAM poster Present 2017;30:35.

5. Kendrick KR, Baxi SC, Smith RM. Usefulness of the modified 0-10 Borg scale in assessing the degree of dyspnea in patients with COPD and asthma. J Emerg Nurs 2000;26:216-22.

6. Parone D, Stauss M, Reed CR, et al. A comparative study of two nebulizers in the emergency department: Breathactuated nebulizer and handheld nebulizer. J Emerg Nurs 2014;40:131-7.
7. Agency for healthcare research and quality. Emergency Severity Index (ESI): A Triage tool for Emergency Departments. [Online]. Available online: https://www. ahrq.gov/professionals/systems/hospital/esi/index.html

8. Sabato K, Ward P, Hawk W, et al. Randomized controlled trial of a breath-actuated nebulizer in pediatric asthma patients in the emergency department. Respir Care 2011;56:761-70.

9. Restrepo RD, Dickson SK, Rau JL, et al. An investigation of nebulized bronchodilator delivery using a pediatric lung model of spontaneous breathing. Respir Care 2006;51:56-61.

10. Ari A, Restrepo RD; American Association for Respiratory Care. Aerosol delivery device selection for spontaneously breathing patients: 2012. Respir Care 2012;57:613-26.

11. Saeed H, Mohsen M, Fink JB, et al. Fill volume, humidification and heat effects on aerosol delivery and fugitive emissions during noninvasive ventilation. J Drug Deliv Sci Technol 2017;39:372-8.

12. Wilkinson M, King B, Iyer S, et al. Comparison of a rapid albuterol pathway with a standard pathway for the treatment of children with a moderate to severe asthma exacerbation in the emergency department. J Asthma 2018;55:244-51.

13. Gardiner MA, Wilkinson MH. Randomized Clinical Trial Comparing Breath-Enhanced to Conventional Nebulizers in the Treatment of Children with Acute Asthma. J Pediatr 2019;204:245-9.e2.

14. Fink J, Dhand R. Bronchodilator resuscitation in the emergency department part 1 of 2: Device selection. Respir Care 1999. Available online: https://www.researchgate.net/ publication/264121994_Bronchodilator_Resuscitation in_the_Emergency_Department_Part_1_or_2_Device_ Selection

15. Dolovich MB, Ahrens RC, Hess DR, et al. Device selection and outcomes of aerosol therapy: Evidencebased guidelines: American College of Chest Physicians/ American College of Asthma, Allergy, and Immunology. Chest 2005;127:335-71.

16. Dugernier J, Hesse M, Vanbever R, et al. SPECTCT Comparison of Lung Deposition using a System combining a Vibrating-mesh Nebulizer with a Valved Holding Chamber and a Conventional Jet Nebulizer: a Randomized Cross-over Study. Pharm Res 2017;34:290-300.

17. Dunne RB, Shortt S. Comparison of bronchodilator administration with vibrating mesh nebulizer and standard jet nebulizer in the emergency department. Am J Emerg 
Med 2018;36:641-6.

18. Moody GB, Luckett PM, Shockley CM, et al. Clinical Efficacy of Vibrating Mesh and Jet Nebulizers With Different Interfaces in Pediatric Subjects With Asthma. Respir Care 2020;65:1451-63.

19. Cantu T, Jenkins L. Quality Improvement Project to Compare Vibrating Mesh Nebulizer Therapy With Hour Long Jet Nebulizer Therapy for Albuterol Delivery in Asthma and Reactive Airway Disorder Patients in a Pediatric Emergency Department. Respir Care 2019;64:3238888.

20. Chweich H, Idrees N, Rice L, et al. Effectiveness of a Vibrating Mesh Aerosolizer Compared to a Jet Nebulizer for the Delivery of Bronchodilator Therapy to Acute Adult Asthmatics in the Emergency Department a Randomized ControlledTrial. Am J Respir Crit Care Med 2019;199:A2209.

21. Carragher RA, Daly CL, Belazarian LA, et al. A Prospectively Designed Chart Review Comparison of Vibrating Mesh Nebulizer Versus Standard Jet Nebulizer to Deliver Bronchodilators in Asthma and COPD Patients in the Emergency Department. Respir Care 2019;64:3227278.

22. Available online: https://m.pdr.net/Mobile/pages/drugsummary/Proventil-HFA-albuterol-sulfate-381

23. Fink J, Dhand R. Bronchodilator resuscitation in the emergency department. Part 2 of 2: dosing strategies. Respir Care 2000;45:497-512.

24. Arnold DH, Moore PE, Abramo TJ, et al. The Dilemma of Albuterol Dosing for Acute Asthma Exacerbations in Pediatric Patients. Chest 2011;139:472.

25. Duarte AG, Dhand R. Chapter 10 Clinical application of aerosols in hospitalized patients. 10.1 Aerosol therapy in the emergency department. The International Society for Aerosols in Medicine Textbook. Available online: https:// www.isam.org/store-textbook/chapter-10

26. Mcfadden ER Jr, Strauss L, Hejal R, et al. Comparison of two dosage regimens of albuterol in acute asthma. Am J Med 1998;105:12-7.

27. Emerman CL, Cydulka RK, McFadden ER. Comparison of $2.5 \mathrm{vs} 7.5 \mathrm{mg}$ of inhaled albuterol in the treatment of acute asthma. Chest 1999;115:92-6.

Cite this article as: Dailey PA, Shockley CM. Review of aerosol delivery in the emergency department. Ann Transl Med 2021;9(7):591. doi: 10.21037/atm-20-4724
28. Muchão FP, Souza JM, Torres HC, et al. Albuterol via metered-dose inhaler in children: Lower doses are effective, and higher doses are safe. Pediatr Pulmonol 2016;51:1122-30.

29. Rodrigo C, Rodrigo G. Therapeutic Response Patterns to High and Cumulative Doses of Salbutamol in Acute Severe Asthma. Chest 1998;113:593-8.

30. Strauss L, Hejal R, Galan G, et al. Observations on the effects of aerosolized albuterol in acute asthma. Am J Respir Crit Care Med 1997;155:454-8.

31. Papo MC, Frank J, Thompson AE. A prospective, randomized study of continuous versus intermittent nebulized albuterol for severe status asthmaticus in children. Crit Care Med 1993;21:1479-86.

32. Khine H, Fuchs SM, Saville AL. Continuous vs intermittent nebulized albuterol for emergency management of asthma. Acad Emerg Med 1996;3:1019-24.

33. Camargo CA Jr, Spooner CH, Rowe BH. Continuous versus intermittent beta-agonists in the treatment of acute asthma. Cochrane Database Syst Rev 2003;(4):CD001115.

34. Gulley SL, Baltzley SM, Junkins AD, et al. Sterility and Stability Testing of Preservative-free Albuterol. J Pediatr Pharmacol Ther 2019;24:53-7.

35. Orth LE, Kelly BJ, Lagasse CA, et al. Safety and effectiveness of albuterol solutions with and without benzalkonium chloride when administered by continuous nebulization. Am J Health Syst Pharm 2018;75:1791-7.

36. Pertzborn MC, Prabhakaran S, Abu-Hasan M, et al. Continuous Albuterol With Benzalkonium in Children Hospitalized With Severe Asthma. Pediatrics 2020;145:e20190107.

37. Griffiths B, Ducharme FM. Combined inhaled anticholinergics and short-acting beta2-agonists for initial treatment of acute asthma in children. Cochrane Database Syst Rev 2013;(8):CD000060.

38. Available online: https://m.pdr.net/Mobile/Pages/drugsummary/Atrovent-HFA-ipratropium-bromide-1743

39. Kirkland SW, Vandenberghe C, Voaklander B, et al. Combined inhaled beta-agonist and anticholinergic agents for emergency management in adults with asthma. Cochrane Database Syst Rev 2017;1:CD001284. 